\title{
Cytoplasmic factors that control nuclear behavior in mammalian oocytes: A re-evaluation of studies performed as a student of Yoshio Masui
}

\author{
Hugh J Clarke \\ Departments of Obstetrics \& Gynecology and Biology, McGill University, Room F3-50, Women's Pavilion, \\ Royal Victoria Hospital, 687 Pine Ave. W., Montreal, Quebec, Canada H3A 1A1
}

Studies performed by the author in the laboratory of Dr Yoshio Masui are reviewed and interpreted in the light of subsequent findings. The first series of studies indicated that that chromosome condensation during meiotic maturation of mouse oocytes is controlled initially by a stable protein that decays during maturation and subsequently by an unstable protein synthesized after germinal vesicle breakdown. Cyclin B is present in immature oocytes, becomes partially degraded near metaphase I and then re-accumulates, suggesting that this may be protein whose activity was inferred from the original results. The second series of experiments indicated that factors which appear in the oocyte cytoplasm during maturation are able to remodel the sperm into metaphaselike chromosomes, and that the supply of these factors is limited. Recent work indicates that these factors are required for the assembly of histones onto the sperm DNA, and has identified two molecular species, mNAP-1 and NPM-3, known to promote replication-independent chromatin assembly in somatic cells, that are expressed in oocytes. (C Elsevier, Paris)

oocyte / meiotic maturation / cell cycle / histones / sperm chromatin

I was introduced to Yoshio Masui through two undergraduate embryology courses that he taught in the Department of Zoology at the University of Toronto, Canada. Through his lectures and selection of research papers to be studied, Yoshio kindled my interest in embryology and especially in the life of the oocyte. I subsequently worked for him for a year as a research assistant and then entered graduate school in the fall of 1978.

The late 1970 s was a busy time in the Masui laboratory. The lab comprised two branches, focused on frog and mouse oocytes, respectively. Peter Meyerhof, who published three seminal papers on the characterization of cytostatic factor (CSF), was the senior graduate student. Manfred Lohka was beginning his work that would lead to developing the cell-free system for studying nuclear behavior during the cell cycle. Soon to join the lab was Ellen Shibuya, who would continue the work on CSF. Leading the mouse work was John
Brazill, who was extending Yoshio's work on DNA repair activity during oocyte meiotic maturation. Margaret Miller, who went on to do graduate work with Yoshio, was a research assistant working on sperm-oocyte interaction. A few years later, Hanna Balakier, already well-known through her studies with Prof. Andrzej Tarkowski on nucleo-cytoplasmic interactions in oocytes using techniques of cell fusion, joined the Masui group.

Yoshio proposed that I join the mouse side of the lab. Gregorio Siracusa and colleagues had recently published a paper showing that mouse eggs at metaphase II could be parthenogenetically activated by an inhibition of protein synthesis (Siracusa et al, 1978). Previous work from Paul Wassarman and others (Stern et al, 1972; Wassarman et al, 1976) had shown that, when germinal vesicle (GV)-stage oocytes were exposed to such inhibitors, they would undergo germinal vesicle breakdown (GVBD) but did not progress beyond metaphase I. 
Yoshio proposed that it would interesting to examine how the response of the oocyte changed during meiotic maturation. First, however, he wrote to Dr Siracusa to inquire whether he was pursuing similar experiments and to ask permission for us to undertake this work. This example provided an early insight into Yoshio's integrity and his perception of the close tie that exists between the individual scientist and his/her research.

I obtained GV-stage oocytes, cultured these to allow meiotic maturation to occur and, at different stages of maturation, exposed them to a protein synthesis inhibitor, puromycin, for $6 \mathrm{~h}$. I examined the morphology of the chromatin at the end of the drug treatment and after protein synthesis had resumed (Clarke and Masui, 1983). I observed that when protein synthesis was inhibited in oocytes that had just undergone GVBD, the chromosomes remained condensed as at metaphase. This resembled the behavior previously reported in oocytes treated at the GV stage. But when protein synthesis was inhibited beginning a few hours later during maturation, at about metaphase I, the oocytes completed the first meiotic division and the chromosomes then decondensed to form a nucleus. This defined a transition point in early maturation, beyond which protein synthesis inhibition caused the oocyte to enter an interphase-like condition.

The behavior of oocytes treated at metaphase I with puromycin resembled that observed by Siracusa et al who had applied the inhibitor at metaphase II; meiotic division was completed after which the oocyte entered interphase. When I removed the metaphase I-treated oocytes from puromycin, however, an unexpected event occurred. They did not undergo DNA replication or mitotic cleavage division, as occurred in oocytes treated at metaphase II. Instead, between 6 and $9 \mathrm{~h}$ after removal of the drug, the chromosomes recondensed and assumed a morphology resembling that observed at meiotic metaphase. Thus, although inhibition of protein synthesis at metaphase I caused oocytes to enter interphase, it did not parthenogenetically activate them. This defined a second transition point, during late maturation, when oocytes became capable of parthenogenetic activation in response to protein synthesis inhibition.

Yoshio and I proposed that the first transition point reflected a change in the properties of the proteins supporting chromosome condensation. According to this model, immature oocytes contained a pool of a relatively stable protein that, when maturation was initiated, promoted chromosome condensation. This protein decayed during maturation, however, and chromosome condensation was then maintained by an unstable protein synthesized sometime after GVBD. Thus, when protein synthesis was inhibited during early maturation, the chromosomes remained condensed because the stable first protein had not yet decayed, and when it was inhibited at metaphase II, they decondensed once the unstable maintenance protein had become depleted. Inhibition of protein synthesis near metaphase I led to chromosome decondensation owing to the natural loss of the stable initiator protein coupled with absence of synthesis of the unstable maintenance protein. In the case of the second transition, we suggested that, relatively late during maturation, oocytes produced a protein that stabilized the decondensed state of the chromatin, and this prevented a return to metaphase once protein synthesis had resumed.

Many of the proteins that govern the cell-cycle transitions of maturation have now been identified, and this model can now be interpreted at a molecular level. Briefly, fully grown immature oocytes contain the components of MPF, p34 and cyclin B (Choi et al, 1991; Chesnel and Eppig, 1995; Mitra and Schultz, 1996; de Vantéry et al, 1996), as well as the $\mathrm{cdc}^{25 \mathrm{C}}$ gene product (Mitra and Schultz, 1996) which may control MPF activation when maturation begins. Immature oocytes also contain mos protein (Paules et al, 1989), which is also synthesized during maturation (Verlhac et al, 1996), and the MAP kinases, $\mathrm{p} 42^{\text {erk2 }}$ and $\mathrm{p} 44^{\text {erk1 } 1}$ (Sobajima et al, 1993; Verlhac et al, 1993), whose phosphorylation and kinase activity during maturation require mos (Verlhac et al, 1996; Hirao and Eppig, 1997a). Near metaphase I, a portion of the cyclin B is degraded (Hampl and Eppig, 1995) and MPF activity declines (Choi et al, 1991; Kubiak et al, 1992; Gavin et al, 1994). By contrast, the quantity of p34 appears to remain constant during this time (Choi et al, 1991). As cyclin B is present in immature oocytes and persists until metaphase I, it may be the stable protein of our model.

After meiosis I, cyclin B re-accumulates due to new synthesis (Hampl and Eppig, 1995) and MPF activity increases. Interestingly, the cyclin $B$ in metaphase II oocytes is less stable than the cyclin B in metaphase I oocytes (Hampl and Eppig, 1995). Cyclin $B$ is continuously synthesized and degraded in oocytes at metaphase II (Kubiak et al, 1993) and declines rapidly in metaphase II oocytes treated with protein synthesis inhibitors (Moos et al, 1996). Furthermore, cyclin B fails to re-accumulate in oocytes in which mos protein has been depleted by injection of antisense oligonucleotides, suggesting that mos may be required to stabilize newly synthesized cyclin B (O'Keefe et al, 1991). When cyclin B re-accumulation is inhibited, it might be predicted that MPF activity would remain low. Consistent with this prediction, oocytes injected with mos antisense oligonucleotides often enter interphase fol- 
lowing meiosis I (O'Keefe et al, 1989, 1991; Gebauer et al, 1994), exactly as occurs following protein synthesis inhibition near metaphase I. On the other hand, in mos- ${ }^{--}$oocytes, MPF is reactivated after metaphase I and the oocytes enter metaphase II (Verlhac et al, 1986); it will be important to know whether cyclin B re-accumulates in these oocytes. Based on these results, we may tentatively propose that the cyclin B synthesized after meiosis I may be the unstable maintenance protein of our model.

It should be emphasized that the relationship between mos, cyclin $B$, and MPF activity remains to be fully explained. When mos is depleted from maturing oocytes, different responses have been observed. Injection of antisense oligonucleotides at the GV stage can lead either to arrest at metaphase I with condensed chromosomes (Paules et al, 1989), or to completion of the first meiotic division followed by entry into interphase (O'Keefe et al, 1989, 1991; Gebauer et al, 1994; Hirao and Eppig, 1997a). In the mos- $^{-1-}$ mice, an even greater range of responses has been observed (Colledge et al, 1994; Hashimoto et al, 1994; Araki et al, 1996; Verlhac et al, 1996; Hirao and Eppig, 1997b). Some oocytes complete meiosis I and then enter interphase, whereas others complete both meiosis I and II and then enter interphase. This may reflect the fact that, although a portion of the cyclin B is degraded at metaphase I, a substantial amount remains (Hampl and Eppig, 1995). Perhaps this amount is close to the threshold required to activate MPF so that differences in mouse genetic background or experimental procedure, which might lower or raise the threshold, determine whether following meiosis I the oocyte enters meiosis II or interphase. In still other cases, however, the oocytes complete both meiotic divisions, enter interphase but then rapidly return to a meiotic metaphase-like condition termed metaphase III (Kubiak, 1989; Verlhac et al, 1996). These oocytes also display unusual microtubular arrays, which may indicate that mos has numerous intracellular targets.

What of the second transition point, beyond which the oocyte remains in interphase after protein synthesis resumes. It seems likely that the metaphase I-treated oocytes, upon withdrawal of puromycin, synthesize cyclin B and mos and consequently return to a meiotic metaphase-like state. Why do metaphase II-treated oocytes not also return to meiotic metaphase? We may suppose that between metaphases I and II, the oocyte acquires some property so that when it exits from metaphase, either by an activating stimulus or by depletion of MPF through protein synthesis inkibition, it enters the mitotic cell cycle. One could further speculate that those oocytes which, when given a parthenogenetic stimulus at metaphase II, complete the second meiotic division and then enter metaphase III (Kubiak, 1989) rather than interphase, have failed to acquire this property.

The nature of this acquired property is entirely speculative. Immature oocytes contain a large stock of mRNAs that are translated during maturation, however, and as in the frog (Sheets et al, 1994) different $m R$ NAs may be translated at different times. It may be hypothesized that proteins appearing at a late stage of maturation confer the ability to enter the mitotic cell cycle. One possibility is that these proteins might degrade mos, provided MPF activity is low (Weber et al, 1991). Thus, depletion of MPF activity at metaphase I would not trigger mos degradation because the components of the proteolytic system had not yet been synthesized. When protein synthesis resumed, the existing mos would stabilize the newly synthesized cyclin B, leading to increased MPF activity and a return to metaphase. In contrast, depletion of MPF activity at metaphase II after these components had been synthesized would trigger mos degradation. In the absence of mos, cyclin B could not accumulate and the oocytes would remain in interphase.

A second possibility is that these proteins might be required for mitotic cell-cycle progression. Cyclin E, which is required for entry into S-phase (Sauer and Lehner, 1995; Reed, 1997), is synthesized during maturation in the frog (Chevalier et al, 1996) and its mRNA is present in mouse oocytes (Moore et al, 1996). Perhaps translation of cyclin $\mathrm{E}$ enables oocytes to enter S-phase and this in turn precludes return to meiotic metaphase. Some support for this notion may be found in experiments in which we induced metaphase I oocytes to enter interphase and then prevented recondensation to metaphase by incubating the oocytes in dibutyryl cyclic AMP (dbcAMP; Clarke and Masui, 1985). Under these conditions, the oocytes began DNA replication about $20 \mathrm{hr}$ later, and some of these developed to the blastocyst stage (Clarke et al, 1988). It could be proposed that normally cyclin E mRNA is translated relatively late during maturation. In oocytes treated at metaphase I with puromycin, translation would not occur until during the post-puromycin incubation in dbcAMP. Once the cyclin $\mathrm{E}$ had been synthesized, the oocytes, being in interphase, would begin DNA replication. This model, however, does not address the possible importance during embryogenesis of the calcium transients that are observed following fertilization but may not occur during 'activation' by protein synthesis inhibition. It would be interesting to test whether mos -1 oocytes, which develop parthenogenetically in the apparent absence of a calcium-releasing trigger, can support normal embryogenesis following appropriate nuclear transfers to provide a paternal genome. 
Following this M.Sc. work, I continued under Yoshio's supervision as Ph.D. candidate. This research work took a different orientation towards the cytoplasmic control of nuclear behavior, namely, the effect of oocyte cytoplasm on nuclei obtained from a different cell type. This conceptual approach was already well established in the Masui laboratory. A previous student, David Ziegler, had published a series of papers describing the behavior of brain nuclei exposed to frog oocyte cytoplasm (Ziegler and Masui, 1973, 1976). As well, Tarkowski and collaborators were performing similar studies in the mouse, using cell fusion to introduce heterologous nuclei into oocytes (Tarkowski and Balakier, 1980; Czolowska et al, 1984). The results of these experiments together with the studies of cultured mammalian cells (Rao and Johnson, 1970; Johnson and Rao, 1970, Sunkara et al, 1979) were central in establishing the concept that very similar cytoplasmic proteins control nuclear behavior in many different cell types.

I examined the effects of oocyte cytoplasm on sperm nuclei (Clarke and Masui, 1986, 1987). Oocytes inseminated during meiotic maturation were not activated by sperm penetration but rather, at least when only one or two sperm entered the oocyte, were able to complete maturation and become arrested at metaphase II. The failure of the oocytes to be activated was probably due to the immaturity of calcium-releasing systems that develop during maturation (Ducibella et al, 1990; Ducibella and Buetow, 1994; Mehlmann and Kline, 1994; Mehlmann et al, 1995; Jones et al, 1995). It meant, however, that we could examine the behavior of the sperm nuclei in a metaphase cytoplasmic environment.

When the inseminated oocytes were maintained in dbcAMP so that they remained arrested at the GV stage, no changes in the sperm nucleus were observed, likely owing in part to the inability of the immature oocyte to reduce the disulfide bridges of the protamines (Miller and Masui, 1982). Once the oocytes underwent GVBD, however, the sperm nucleus underwent a characteristic sequence of morphological changes. Initially, the chromatin became dispersed within the ooplasm. By several hours later, it had condensed into a smaller mass. Following further incubation, this mass frequently became resolved into twenty individual chromosomes. Thus, the oocyte possessed a capacity to organize the highly condensed protamine-containing chromatin of the sperm into chromosome-like structures in the absence of pronuclear formation or DNA replication.

Three points emerged from this study. First, the initial dispersion and condensation of the sperm chromatin resembled the early morphological changes observed following normal fertilization at metaphase II (Ecklund and Levine, 1975; Wright and Longo, 1988). This suggested that the underlying molecular changes might be similar, despite differences between the cytoplasms of unactivated and activated oocytes; for example, the existence of high and low MPF activity, respectively. Second, a maximum of three or four sperm could be remodelled to metaphasic chromosomes within the cytoplasm of a single oocyte, and this number could be doubled when two oocytes were fused together and decreased when oocytes were bisected. This suggested that the re-organization of the sperm chromatin required an oocyte component whose supply was limiting. Third, when the GV was removed from oocytes before insemination, they were unable to remodel the sperm into metaphasic chromosomes. Thus, oocyte nuclear factors were required for this process.

In my laboratory, we have begun to try to identify the oocyte factors that interact with the sperm chromatin and mediate its remodeling into metaphasic chromosomes. The rationale is that the same factors will likely also control remodeling into the male pronucleus following normal fertilization at metaphase II. To this end, however, it was important first to establish that the remodeling of sperm chromatin in maturing oocyte cytoplasm produced physiologically relevant chromatin. Since extensive biochemical analysis is not feasible in the mouse system, we have analyzed properties of the remodeled sperm chromatin. Wafa Harrouk (Harrouk and Clarke, 1993) showed that a microtubular spindle typically became associated with remodeled sperm chromatin, but never with sperm chromatin that had dispersed but failed to recondense. This implied that remodeling had altered sperm chromatin in a way that made it competent to attract or interact appropriately with microtubules. David McLay (McLay and Clarke, 1997) later showed that sperm chromatin remodeled in metaphase oocyte cytoplasm would, upon parthenogenetic activation of the oocyte, form a pronucleus and undergo DNA replication. This provided further evidence that the remodeling of sperm chromatin into metaphasic chromosomes in maturing oocyte cytoplasm produces a physiologically relevant structure.

We have recently begun to study the molecular basis of these morphological changes (McLay and Clarke, 1997). Using immunofluorescence, protamines can be detected on the dispersed sperm chromatin but not on the recondensed chromatin. By contrast, histones $\mathrm{H} 2 \mathrm{~B}$ and $\mathrm{H} 3$ are detectable only on the recondensed sperm chromatin. Thus, recondensation appears to be a morphological marker, indicating that the sperm chromatin has been organized into a somatic-like structure. When 
protein synthesis is inhibited during maturation, the chromatin remains in the dispersed condition and neither $\mathrm{H} 2 \mathrm{~B}$ nor $\mathrm{H} 3$ are detectable. Thus, assembly of these histones onto sperm chromatin in the maturing oocyte cytoplasm requires protein synthesis.

Is it the histones themselves or proteins required to assemble the histones into chromatin that need to be made by the maturing oocyte? To study this question, we injected calf thymus histones into oocytes and then inseminated them in the presence of puromycin. Despite the presence of exogenous histones, the sperm chromatin remained dispersed and only a weak association of histones with the chromatin was observed. These results favor the idea that proteins that assemble the histones into chromatin need to be synthesized during maturation. They do not rule out that the histone also must be synthesized during maturation; however, it may be noted that histone $\mathrm{H} 4$ accumulates in growing oocytes prior to maturation (Wassarman and Mrozak, 1981).

One possibility is that these putative histoneassembly proteins are functionally similar to nucleoplasmin, which mediates protamine-histone exchange in amphibian oocytes (Dilworth et al, 1987; Ohsumi and Katagiri, 1991; Philpott et al, 1991). Nucleoplasmin has not yet been identified in mammalian oocytes. By RT-PCR analysis of growing and fully grown oocytes, we have identified mRNAs corresponding to two nucleoplasminrelated proteins, known as mNAP-1 (Ito et al, 1996) and NPM-3 (MacArthur and Shackleford, 1997) (McLay and Clarke, unpublished). It is possible that these help to assemble the histones onto the paternal DNA, both in the maturing oocyte and after normal fertilization at metaphase II. We are currently testing whether the mRNAs encoding mNAP-1 and NPM-3 are translated during maturation and whether their depletion will prevent sperm chromatin remodelling in unactivated and activated oocyte cytoplasms.

These results suggest that, during meiotic maturation, oocytes synthesize the molecules that will be used after fertilization to remove the protamines from the sperm chromatin and assemble histones and non-histone chromosomal proteins onto the paternal DNA to produce functional chromatin. Once present in the cytoplasm, these factors can function independently of whether the oocyte has been activated by fertilization. Thus, when sperm chromatin is exposed either to unactivated or to activated oocyte cytoplasm, its nuclear membrane is removed and the protamines are removed as the chromatin disperses. Histones and other chromatin components are subsequently assembled onto the DNA and this chromatin recondenses into a smaller mass. Only the final step in the remodeling process depends on the cell-cycle state of the oocyte - in unactivated oocytes, high MPF activity promotes resolution of the recondensed mass into individual metaphase chromosomes; in activated oocytes, the absence of MPF permits its decondensation to form the male pronucleus.

Understanding how oocyte cytoplasmic components interact with nuclear elements will receive even more attention following the recent reports that mammals can be cloned using differentiated cell nuclei (Campbell et al, 1996, Wilmut et al, 1997, Wakayama et al, 1998). Although mammalian cloning is currently very inefficient, it is has been observed that serial nuclear transplantations, whose effect is to expose the nuclei repeatedly to oocyte cytoplasm, significantly increase the frequency of development. Earlier studies in amphibians had similarly indicated that conditioning of differentiated nuclei in oocyte cytoplasm enhanced their ability to support subsequent embryonic development (DeBerardino, 1980). These results strongly suggest that the structure or composition of the transplanted nuclei are altered during their residence in the oocyte cytoplasm. But we know almost nothing of these changes. What proteins, if any, are added or removed? Do these changes make the nucleus totipotent? Do specific molecular characteristics exist that define the totipotent state? A more complete understanding of the biochemical modifications that occur in foreign nuclei exposed to oocyte cytoplasm will be necessary before these questions may be answered.

In closing, I would like to reflect upon qualities of Yoshio Masui as a supervisor. I believe that his primary interest was in nourishing the intellectual development of the members of his laboratory. There was no single, large laboratory project, to which individuals contributed small components. Rather, Yoshio assigned separate projects, and expected each student to work alone and to be responsible for each aspect of the project. This approach meant also that we were given considerable freedom to decide the orientation of the project. Of course, he challenged us with questions about the details of our experiments. But, none of these questions appropriated the project, which remained with the student. In addition, Yoshio was ready to spend his time freely working with us, whether planning experiments, editing a manuscript, or discussing a recent paper. From the perspective of the student taking the first steps towards a career in scientific research, what he provided was a sense of importance. That each student worked independently inculcated in us a sense of responsibility for the success of our project and, when it reached completion, of achievement. 
Yoshio Masui as a supervisor also emphasized the need to develop and practice scientific skepticism: to try to look beyond the details of the experiments to a philosophical analysis of the assumptions that underlay the experimental design and of the logical implications of the interpretations. Yoshio regularly demonstrated how one could bring a set of reasonable assumptions, different from the authors', to evaluate an experimental result and use these to arrive at a conclusion quite different from that proposed by the authors. I believe that Yoshio wanted us to learn to uncover our hidden assumptions, and to understand how powerfully these assumptions could direct the experiments we did and limit how we interpreted the results. As I now supervise my own graduate students, I often reflect on and try to apply these qualities of Yoshio Masui as a supervisor. But beyond this teaching and training, I hope that I can instill in my students the same fascination with the egg that Yoshio has given to me.

\section{REFERENCES}

Araki K, Naito K, Haraguchi S, Suzuki R, Yokoyama M, Inoue M, Aizawa S, Toyoda Y and Sato E (1996) Meiotic abnormalities of c-mos knockout mouse oocytes: activation after first meiosis or entrance into third meiotic metaphase. Biol Reprod 55, 1315-1324

Chesnel F and Eppig JJ (1995) Synthesis and accumulation of p34cdc2 and cyclin B in mouse oocytes during acquisition of competence to resume meiosis. Mol Reprod Dev 40, 503-508

Chevalier S, Couturier A, Chartrain I, I e Guellec R, Beckhelling C, Le Guellec K, Philippe M and Ford CC (1996) Xenopus cyclin $E$, a nuclear phosphoprotein, accumulates when oocytes gain the ability to initiate DNA replication. / Cull Sci 109, 1173-1184

Choi T, Aoki F, Mori M, Yamashita M, Nagahama $Y$ and Kohmoto $K$ (1991) Activation of $p^{34}{ }^{\text {cdc2 }}$ protein kinase activity in meiotic and mitotic cell cycles in mouse oocytes and embryos. Development 113,789-795

Campbell KH, McWhir J, Ritchie WA and Wilmut I (1996) Sheep cloned by nuclear transfer from a cultured cell line. Nature 380, 64-66

Clarke HI and Masui $Y(1983)$ The induction of reversible and irreversible chromosome decondensation by protein synthesis inhibition during meiotic maturation of mouse oocytes. Dev Biol 97, 291-301

Clarke HJ and Masui $Y$ (1985) Inhibition by dibutyryl cyclic AMP of the transition to metaphase of mouse oocyte nuclei and its reversal by cell fusion to metaphase oocytes. Dev Biol 108, 32-37

Clarke HJ, and Masui $Y$ (1986) Transformation of sperm nuclei to metaphase chromosomes in the cytoplasm of maturing oocytes of the mouse. I Cell Biol 102, 1039-1046

Clarke HJ and Masui Y (1987) Dose-dependent relationship between oocyte cytoplasmic volume and transformation of sperm nuclei to metaphase chromosomes. J Cell Biol 104, 831-840

Clarke HI, Rossant I and Masui Y (1988) Suppression of chromosome condensation during meiotic maturation induces parthenogenetic development of mouse oocytes. Development $104,97-103$

Colledge WH, Carlton MBL, Udy GB and Evans MJ (1994) Disruption of c-mos causes parthenogenetic development of unfertilized mouse eggs. Nature 370, 65-68

Czolowska R, Modlinski JA and Tarkowski AK (1984) Behavi- our of thymocyte nuclei in nonactivated and activated mouse oocytes. J Cell Sci 69, 19-34

de Vantéry C, Gavin AC, Vassalli JD and Schorderet-Slatkine S (1996) An accumulation of $\mathrm{p}^{34^{\mathrm{dc}} 2}$ at the end of mouse oocyte growth correlates with the acquisition of meiotic competence. Dev Biol 174, 335-44

DiBerardino MA (1980) Genetic stability and modulation of metazoan nuclei transplanted into eggs and zygotes. Differentiation $17,17-30$

Dilworth SM, Black SJ and Laskey RA (1987) Two complexes that contain histones are required for nucleosome assembly in vitro: role of nucleoplasmin and N1 in Xenopus egg extracts. Cell 51, 1009-1018

Ducibella T, Duffy P. Reindollar R and Su B (1990) Changes in the distribution of mouse oocyte cortical granules and ability to undergo the cortical reaction during gonadotropinstimulated meiotic maturation and aging in vivo. Biol Reprod $43,870-876$

Ducibella T, and Buetow J (1994) Competence to undergo normal, fertilization-induced cortical activation develops after metaphase I of meiosis in mouse oocytes. Dev Biol 165, 95104

Ecklund PS and Levine L (1975) Mouse sperm basic nuclear protein: electrophoretic characterization and fate after fertilization. J Cell Biol 66, 251-262

Gavin AC, Cavadore JC and Schorderet-Slatkine S (1994) Histone $\mathrm{H} 1$ kinase activity, germinal vesicle breakdown and $\mathrm{M}$ phase entry in mouse oocytes. J Cell Sci 107, 275-283

Gebauer F, Xu W, Cooper GM and Richter JD (1994) Translational control by cytoplasmic polyadenylation of c-mos mRNA is necessary for oocyte maturation in the mouse. EMBO / 13, 5712-5720

Hampl A, and Eppig JJ (1995) Analysis of the mechanism(s) of metaphase I arrest in maturing mouse oocytes. Development $121,925-933$

Harrouk W and Clarke HJ (1993) Sperm chromatin acquires an activity that induces microtubule assembly during residence in the cytoplasm of metaphase oocytes of the mouse. Chromosoma 102, 279-86

Hashimoto N, Watanabe N, Furuta Y, Tamemoto H, Sagata N, Yokoyama M, Okazaki K, Nagayoshi M, Takeda N, Ikawa $Y$ and Aizawa $S$ (1994) Parthenogenetic activation of oocyles in c-mos-deficient mice. Nature 370, 68-71

Hirao Y and Eppig JJ (1997) Analysis of the mechanism(s) of metaphase I arrest in strain LT mouse oocytes: participation of MOS. Development 124, 5107-5113

Hirao X and Eppig JJ (1997) Parthenogenetic development of Mos-deficient mouse oocytes. Mol Reprod Dev 48, 391-396

Ito T, Bulger M, Kobayashi R and Kadonaga JT (1996) Drosophila NAP-1 is a core histone chaperone that functions in ATP-facilitated assembly of regularly spaced nucleosomal arrays. Mol Cell Biol 16, 3112-3124

Johnson RT and Rao PN (1970) Mammalian cell fusion: Induction of premature chromosome condensation in interphase nuclei. Nature 226, 717-722

Jones KT, Carroll J and Whittingham DG (1995) Ionomycin, thapsigargin, ryanodine, and sperm induced $\mathrm{Ca}^{2+}$ release increase during meiotic maturation of mouse oocytes. I Biol Chem 270, 6671-6677

Kubiak JZ (1989) Mouse oocytes gradually develop the capacity for activation during the metaphase II arrest. Dev Biol $136,537-545$

Kubiak JZ, Weber M, Géraud G and Maro B (1992) Cell cycle modification during the transition between meiotic $M$ phases in mouse oocytes. J Cell Sci 102, 457-467

Kubiak JZ, Weber M, de Pennart H, Winston N and Maro B (1993) The metaphase II arrest in mouse oocytes is controlled through microtubule-dependent destruction of cyclin B in the presence of CSF. EMBO J 12, 3773-3778

MacArthur CA and Shackleford GM (1997) Npm3: a novel, widely expressed gene encoding a protein related to the molecular chaperones nucleoplasmin and nucleophosmin. Genomics 42, 137-140

McLay DW and Clarke HJ (1997) The ability to organize sperm 
DNA into functional chromatin is acquired curing meiotic maturation in murine oocytes. Dev Biol 186, 73-84

Mehlmann LM and Kline D (1994) Regulation of intracellular calcium in the mouse egg: calcium release in response to sperm or inositol trisphosphate is enhanced after meiotic maturation. Biol Reprod 51, 1088-1098

Mehlmann LM, Terasaki M, Jaffe LA and Kline D (1995) Reorganization of the endoplasmic reticulum during meiotic maturation of the mouse oocyte. Dev Biol 170, 607-615

Miller MA and Masui Y (1982) Changes in the stainability and sulfhydryl level in the sperm nucleus during sperm-oocyte interaction in mice. Gamete Res 5, 167-179

Mitra J and Schultz RM (1996) Regulation of the acquisition of meiotic competence in the mouse: changes in the subcellular localization of $\mathrm{cdc}^{2}$, cyclin B1, $\mathrm{cdc}^{25 \mathrm{C}}$ and wee', and in the concentration of these proteins and their transcripts. J Cell Sci 109, 2407-2415

Moore GD, Ayabe T, Kopf GS and Schultz RM (1996) Temporal patterns of gene expression of G1-S cyclins and cdks during the first and second mitotic cell cycles in mouse embryos. Mol Reprod Dev 45, 264-275

Moos J, Kopf GS and Schultz RM (1996) Cycloheximideinduced activation of mouse eggs: effects on cdc2/cyclin B and MAP kinase activities. J Cell Sci 109, 739-748

Ohsumi K and Katagiri C (1991) Characterization of the ooplasmic factor inducing decondensation of and protamine removal from toad sperm nuclei: involvement of nucleoplasmin. Dev Biol 148, 295-305

O'Keefe SJ, Wolfes H, Kiessling AA and Cooper GM (1989) Microinjection of antisense c-mos oligonucleotides prevents meiosis $1 \mathrm{I}$ in the maturing mouse egg. Proc Natl Acad Sci USA 86, 7038-7042

O'Keefe SJ, Kiessling AA and Cooper GM (1991) The c-mos gene product is required for cyclin $B$ accumulation during meiosis of mouse eggs. Proc Natl Acad Sci USA 88, 7869-7872

Paules R, Buccione R, Moschel RC, Vande Woude GF and Eppig JJ (1989) Mouse Mos protooncogene product is present and functions during oogenesis. Proc Natl Acad Sci USA 86, 5395-5399

Philpott A, Leno GH and Laskey RA (1991) Sperm decondensation in Xenopus egg cytoplasm is mediated by nucleoplasmin. Cell 65, 569-578

Rao PN and Johnson RT (1970) Mammalian cell fusion: Studies on the regulation of DNA synthesis and mitosis. Nature 225, 159-164

Reed SI (1997) Control of the G1/S transition. Cancer Survey 29, 7-23

Sauer K and Lehner CF (1995) The role of cyclin E in the regulation of entry into S phase. Progr Cell Cycle Res 1, 125-39

Sheets MD, Fox CA, Hunt T, Vande Woude G and Wickens M. (1994) The 3c-untranslated regions of c-mos and cyclin mRNAs stimulate translation by regulating cytoplasmic polyadenylation. Genes Dev 8, 926-938

Siracusa G, Whittingham DG, Molinaro M and Vivarelli E (1978)
Parthenogenetic activation of mouse oocytes induced by inhibitors of protein synthesis. J Embryol Exp Morphol 43, 157166

Sobajima T, Aoki F and Kohmoto K (1993) Activation of mitogen-activated protein kinase during meiotic maturation in mouse oocytes. J Reprod Fertil 97, 389-394

Stern S, Rayyis A and Kennedy JF (1972) Incorporation of amino acids during maturation in vitro by the mouse oocyte: Effect of puromycin on protein synthesis. Biol Reprod 7, 341-346

Sunkara PS, Wright DA and Rao PN (1979) Mitotic factors from mammalian cells induce germinal vesicle breakdown and chromosome condensation in amphibian oocytes. Proc Natl Acad Sci USA 76, 2799-2802

Tarkowski AK and Balakier H (1980) Nucleo-cytoplasmic interactions in cell hybrids between mouse oocytes, blastomeres and somatic cells. J Embryol Exp Morphol 55, 319-330

Verlhac MH, Kubiak JZ, Weber M, Géraud G, Colledge WH, Evans MJ and Maro B (1996) Mos is required for MAP kinase activation and is involved in microtubule organization during meiotic maturation in the mouse. Development 122, 815-822

Wakayama T, Perry ACF, Zuccotti M, Johnson KR and Yanagimachi R (1998) Full-term development of mice from enucleated oocytes injected with cumulus cell nuclei. Nature 394, 369-374

Wassarman PM, Josefowicz WJ and Letourneau GE (1976) Meiotic maturation of mouse oocytes in vitro: Inhibition of maturation at specific stages of nuclear progression. J Cell Sci 22, 531-545

Wassarman PM and Mrozak SC (1981) Program of early development in the mammal: synthesis and intracellular migration of histone H4 during oogenesis in the mouse. Dev Biol 84, 364-371

Weber M, Kubiak JZ, Arlinghaus RB, Pines J and Maro B (1991) c-mos proto-oncogene product is partly degraded after release from meiotic arrest and persists during interphase in mouse zygotes. Deo Biol 148, 393-397

Wilmut I, Schnieke AE, McWhir J, Kind AJ and Campbell KH (1997) Viable offspring derived from fetal and adult mammalian cells. Nature $385,810-813$

Wright SJ and Longo FJ (1988) Sperm nuclear enlargement in fertilized hamster eggs is related to meiotic maturation of the maternal chromatin. I Exp Zool 247, 155-165

Ziegler D and Masui Y (1973) Control of chromosome behavior in amphibian oocytes. I. The activity of maturing oocytes inducing chromosome condensation in transplanted brain nuclei. Dev Biol 35, 283-292

Ziegler D and Masui Y (1976) Control of chromosome behavior in amphibian oocytes. II. The effect of inhibitors of RNA and protein synthesis on the induction of chromosome condensation. J Cell Biol 68, 620-628

Received 16 August 1998; accepted 12 November 1998 